\title{
Treatment of hypertension
}

\author{
A. E. Doyle \\ From the Department of Medicine, University of Melbourne, Austin Hospital, \\ Heidelberg, Victoria 3084, Australia
}

The aim of treatment of hypertension as of other diseases is to relieve symptoms and to improve prognosis. Most of the symptoms of hypertension are due to the associated cardiovascular complications, and these also have a decisive effect on prognosis.

The effectiveness of treatment for hypertension depends on the extent of reduction of blood pressure, and there is good evidence that consistently lowering the blood pressure may lead to improvement in many symptoms such as headache, paroxysmal nocturnal dyspnoea, and angina of effort, and to a decrease in mortality.

However, all methods of treatment have some disadvantages. Most drugs have some side effects, which range in severity from mild nuisance effects to major complications. Moreover, many drugs are capable of inducing falls of blood pressure so large that transient or permanent tissue ischaemia may develop, especially in patients with severe vascular disease.

For these reasons in the past most of us have considered that not all hypertensive patients should be given active treatment and that only those with either symptoms or an obviously poor prognosis should be treated. This has been necessary because of the need to try to improve symptoms and prognosis without the substitution of severe or disabling side effects. Perhaps the most striking improvement in the effectiveness of drug treatment of hypertension in the last decade has resulted from increased awareness on the part of physicians of the value of combinations of drugs. While many still remain suspicious of drug combinations, this is probably because the successful use of multiple therapeutic agents needs to be soundly based on an understanding of the mechanism of drug actions.

\section{Dose-response relationship}

As an illustration, one can examine the relationship between dose and response in a pharmacologically active drug - for example, a ganglion-blocking drug. Such a drug has both a desired blood pressure lowering effect and an undesired effect on (for example) salivary secretion. When the drug is given alone the dose-response relationships between the desired and undesired effect and dose are identical. This implies that if the drug is used alone the desired therapeutic effect can only be attained at a dose which also causes side effects. If another drug is added, say reserpine, which acts in a different way on the target organ (in this case the salivary glands), the two dose-response curves for the desired and undesired action of the drug are separated to the extent that it becomes possible to attain a useful therapeutic effect at a dose which has few if any unwanted effects.

In specific terms the thiazide diuretics have proved to be the most powerful agents having this potentiation of the cardiovascular effects of hypotensive drugs. It is perhaps worth emphasizing, to an audience mainly concerned with using these agents as diuretics in oedematous states, that their use in hypertension uncomplicated by oedema usually leads to little potassium depletion, at least as far as serum levels of potassium are concerned. The standard dose of chlorothiazide of $0.5 \mathrm{~g}$. twice daily has been consumed regularly by many patients in our clinic for periods of up to ten years without clinical evidence of disability attributable to hypokalaemia. A more serious defect of these drugs in hypertension is the high incidence of hyperuricaemia which they induce. In our series (Mathew et al., 1967) the serum uric acid levels have risen above $7 \mathrm{mg}$./ $100 \mathrm{ml}$. in over half the patients taking thiazide diuretics regularly, and clinical gout has occurred in about 15 per cent. In other series even higher incidences have been noted (Demartini et al., 1962). Although the adverse effects of hyperuricaemia on renal function and its relationship to clinical gout make this a serious drawback to the use of thiazides in the treatment of hypertension, nevertheless the efficiency with which these drugs potentiate the effects of other hypotensive drugs makes them 
the treatment of first choice. They are, however, seldom effective without other therapy.

\section{Alpha methyl dopa}

The second major advance in the last few years has been the introduction of alpha methyl dopa. This substance has a complex mode of action; it almost certainly causes adrenergic terminal depletion of noradrenaline, probably replacing it with the false transmitter $\alpha$-methyl noradrenaline (Day and Rand, 1963), and very probably has a central action as well. Clinically it has the great advantage of a rather shallow dose-response curve, so that sudden episodes of hypotension are unusual and few side effects occur. Its most interesting side effect is the induction of a positive Coombs test of the pure IgG type which occurs in almost $25 \%$ of those taking large doses (Carstairs et al., 1966; Louis et al., 1967). However, the occurrence of haemolytic anaemia is very unusual, even in patients continuing to take large doses some years after a positive Coombs test had first been detected. It seems that as well as being an effective drug methyl dopa has also the distinction of having cast considerable doubt on the concept of autoimmune haemolytic anaemia.

Unfortunately, because methyl dopa is easy to use and has not many very conspicuous side effects, it is often used thoughtlessly and ineffectively. The drug is too often used in a routine way as a rather expensive placebo without careful assessment of the response.

About half of the patients with severe hypertension can be satisfactorily controlled with the combination of methyl dopa and a diuretic. However, even in the remainder who do not achieve satisfactory control of blood pressure with methyl dopa and a diuretic, this combination of drugs serves as a satisfactory one to potentiate other hypotensive agents.

\section{Clonidine and other drugs}

It is our practice now to use clonidine as the next drug to add to the above mentioned agents in resistant hypertensive patients. Clonidine (Catapressan) is an imidazoline derivative closely related chemically to tolazoline. Pharmacological studies have shown that the fall in blood pressure and bradycardia which this drug induces are due to a reduction in sympathetic tone, apparently of central origin, affecting the heart and blood vessels (Randand Wilson I968). When it is givenalone, most patients require doses large enough to cause severe dry mouth and profound sleepiness. Given in smaller doses in combination with diuretics and methyl dopa, side effects are greatly diminished and control of blood pressure is satisfactory in most patients (Ebringer et al., 1970).

There remain a number of patients who need additional drugs. For these, adrenergic blocking agents such as guanethidine or bethanidine or ganglionic blocking drugs such as pempidine still have a place in combination with the other agents.

With most patients it is possible using such combinations of drugs to treat hypertension with comparatively few side effects. These patients tend to form the silent majority of hypertensive patients. There remain, however, a few problem patients of whom, like most consultant physicians, I suspect I receive more than my share.

What can be done for this small minority of difficult patients ? Patients fail to respond to drug treatment for hypertension for several reasons. Perhaps the commonest reason is that they do not take the drugs. A simple fluorimetric analysis of the urine for the presence of large amounts of catecholamines is perhaps the easiest way to check this problem. Very high catechol levels in the urine are always found in patients taking methyl dopa.

\section{Sympathectomy}

Patients who do in fact take their drugs and do not respond, or who have intolerable side effects, need full investigation to exclude the presence of operable causes of hypertension such as renal artery stenosis. Such investigation is worth while although seldom successful. If, as usually happens, no remediable cause is obvious, bilateral thoracolumbar sympathectomy may have to be undertaken. This operation, although not always effective alone, certainly usually increases the sensitivity of the patient to hypotensive drugs. The disadvantages of this are, of course, the reasons which have led to the operation now being seldom performed - namely, operative mortality and postoperative morbidity.

More recently we have been studying the possibility of inducing partial sympathectomy by chemical means. This possibility suggested itself when, during the course of treatment with guanacline, an adrenergic blocking agent resembling guanethidine, six patients developed severe postural hypotension which lasted several weeks (Jerums, Ebringer, and Doyle, 1968). After a few weeks the symptoms abated, but these patients have remained normotensive now for almost eighteen months with no treatment other than diuretics (Dawborn et al., 1969). Animal studies have sub- 
sequently confirmed that the hypotensive effects of this drug may be prolonged. Histological studies of autonomic ganglia show heavy deposits of lipofuscin which persist after guanacline treatment and which presumably represent an end result of injury to the adrenergic terminals. Similar changes occur in human autonomic ganglia in patients treated with guanacline, but their significance in these is less easy to establish because similar changes occur with ageing. A preliminary investigation of the deliberate use of this drug to induce long-lasting sympathetic impairment is yielding promising results in a few difficult patients.

\section{Haemodialysis}

Finally, the treatment of hypertension with severe renal failure has been considerably altered by the development of haemodialysis. Renal failure is a common cause of death in malignant hypertension, and when it is present it may be very difficult to manage. In common with other groups we have found that in such patients bilateral nephrectomy may make control of blood pressure much easier, probably because most such patients in our experience have very high levels of plasma renin and angiotensin.

In summary, most patients with hypertension can be fairly easily controlled with the standard drugs available today. Most difficult patients can be treated, but the methods of treatment for this small group of patients are complex and radical.

The ease with which most patients can currently be treated must inevitably cause revaluation of the indications for treatment in hypertension. In particular, it is necessary to make a decision on whether patients with mild uncomplicated hypertension should now be treated. This decision involves making a guess at whether any long-term disadvantages of drug treatment will outweigh the probable advantages of prevention of the development of cardiovascular complications. Although evidence is incomplete, our policy now is to accept all hypertensive patients, however mild or early, for long-term control of blood pressure.

\section{References}

Carstairs, K. C., Breckenridge, A., Dollery, C. T., and Worlledge, S. M. (1966). Incidence of a positive direct Coombs test in patients on $\alpha$-methyldopa, Lancet, 2, 133.

Dawborn, J. K., Doyle, A. E., Ebringer, A., Howqua, J., Jerums, G., Johnston, C. I., Mashford, M. L., and Parkin, J. D. (1969). Persistent postural hypotension due to Guanacline. Pharmacologia Clinica, 2, I.

Day, M. D., and Rand, M. J. (1963). A hypothesis for the mode of action of $\alpha$-methyldopa in relieving hypertension. Fournal of Pharmacy and Pharmacology, 15, 221 .

Demartini, F. E., Wheaton, E. A., Healey, L. A., and Laragh, J. H. (1962). Effect of chlorothiazide on the renal excretion of uric acid. American fournal of Medicine, 32, 572.

Ebringer, A., Doyle, A. E., Dawborn, J. K., Johnston, C. I., and Mashford, M. L. (1970). Clonidine in the treatment of hypertension. Medical Fournal of Australia, 1, 524.

Jerums, G., Ebringer, A., and Doyle, A. E. (1968). A comparison of guanacline with methyldopa in the treatment of hypertension. Medical fournal of Australia, 2, 466.

Louis, W. J., Doyle, A. E., Jerums, G., and KincaidSmith, P. (1967). Methyldopa and haemolytic anaemia. Medical fournal of Australia, 2, 104.

Mathew, T. H., Kincaid-Smith, P., Doyle, A. E., and Baird, C. W. (1967). A comparison of the hyperuricaemic effect of chlorothiazide and quinethazone. Medical fournal of Australia, 1, 493.

Rand, M. J., and Wilson, J. (r968). Mechanisms of the pressor and depressor action of STI55 (2-(2-6dichlorophenylamine)-2-imidazoline hydrochloride ('Catapres'). European fournal of Pharmacology, 3, 27. 